\title{
Solve fraud detection problem by using graph based learning methods
}

\author{
Loc Tran, Tuan Tran, and An Mai \\ John von Neumann Institute, VNU-HCM, Ho Chi Minh City, Vietnam.
}

\begin{abstract}
The credit cards' fraud transactions detection is the important problem in machine learning field. To detect the credit cards' fraud transactions help reduce the significant loss of the credit cards' holders and the banks. To detect the credit cards' fraud transactions, data scientists normally employ the un-supervised learning techniques and supervised learning technique. In this paper, we employ the graph p-Laplacian based semi-supervised learning methods combined with the under-sampling technique such as Cluster Centroids to solve the credit cards' fraud transactions detection problem. Experimental results show that that the graph p-Laplacian semi-supervised learning methods outperform the current state of art graph Laplacian based semi-supervised learning method $(p=2)$.
\end{abstract}

\section{Key words: graph p-Laplacian, credit card, fraud detection, semi-supervised learning}

\section{Introduction}

While purchasing online, the transactions can be done by using credit cards that are issued by the bank. In this case, if the cards or cards' details are stolen, the fraud transactions can be easily carried out. This will lead to the significant loss of the card holder or the bank. In order to detect credit cards' fraud transactions, data scientists employ a lot of machine learning techniques. To the best of our knowledge, there are two classes of machine learning techniques used to detect credit cards' fraud transactions which are un-supervised learning techniques and supervised learning techniques. The unsupervised learning techniques used to detect credit cards' fraud transactions are k-means clustering technique [1], k-nearest neighbors technique [1], Local Outlier Factor technique [1], to name a few. The supervised learning techniques used to detect credit cards' fraud transactions are Hidden Markov Model technique [2], neural network technique [3], Support Vector Machine technique [4], to name a few.

To the best of our knowledge, the graph based semisupervised learning techniques [5] have not been applied to the credit cards' fraud transactions detection problem. In this paper, we will apply the un-normalized graph pLaplacian based semi-supervised learning technique [6, 7] combined with the under-sampling technique to the credit cards' fraud transactions detection problem.

We will organize the paper as follows: Section 2 will present the initial representations and descriptions used in this paper. Section 3 will present the characterizations of the gradient and divergence operators of graphs.
Section 4 will introduce the characterization of Laplace operator of graphs and its assets. Section 5 will present the characterization of the curvature operator of graphs and its assets. Section 6 will present the characterization of the p-Laplace operator of graphs and its assets. Section 7 will illustrate how to develop the unnormalized graph p-Laplacian based semi-supervised learning method from regularization framework. In section 8, we will compare the exactness of the unnormalized graph Laplacian based semi-supervised learning algorithm (i.e. the current state of art graph based semi-supervised learning method) combined with the under-sampling technique such as Cluster Centroids technique [8] and the un-normalized graph p-Laplacian based semi-supervised learning algorithms combined with Cluster Centroids technique [8]. Section 9 will present the conlusions and discuss the upcoming path of studies.

\section{Preliminary notations and definitions}

Given a graph $G=(V, E, W)$ where $V$ is a set of vertices with $|V|=n, E \subseteq V * V$ is a set of edges and $W$ is a $n *$ $n$ similarity matrix with elements $w_{i j} \geq 0(1 \leq i, j \leq$ $n)$.

Also, please note that $w_{i j}=w_{j i}$.

The degree function $d: V \rightarrow R^{+}$is

$$
d_{i}=\sum_{j \sim i} w_{i j},(1)
$$

where $j \sim i$ is the set of vertices adjacent with $i$.

Define $D=\operatorname{diag}\left(d_{1}, d_{2}, \ldots, d_{n}\right)$.

The inner product on the function space $R^{V}$ is 


$$
<f, g>_{V}=\sum_{i \in V} f_{i} g_{i}(2)
$$

Also define an inner product on the space of functions $R^{E}$ on the edges

$$
<F, G>_{E}=\sum_{(i, j) \in E} F_{i j} G_{i j}(3)
$$

Here let $H(V)=\left(R^{V},<_{.}, .>_{V}\right)$ and $H(E)=\left(R^{E},<\right.$ $\left.\ldots,>_{E}\right)$ be the Hilbert space real-valued functions defined on the vertices of the graph $G$ and the Hilbert space of real-valued functions defined in the edges of $G$ respectively.

\section{Gradient and Divergence Operators}

We define the gradient operator $d: H(V) \rightarrow H(E)$ to be

$$
(d f)_{i j}=\sqrt{w_{i j}}\left(f_{j}-f_{i}\right),(4)
$$

where $f: V \rightarrow R$ be a function of $H(V)$.

We define the divergence operator div: $H(E) \rightarrow H(V)$ to be

$$
<d f, F>_{H(E)}=<f,-\operatorname{div} F>_{H(V)},(5)
$$

where $f \in H(V), F \in H(E)$.

Thus, we have

$$
(\operatorname{divF})_{j}=\sum_{i \sim j} \sqrt{w_{i j}}\left(F_{j i}-F_{i j}\right)(6)
$$

\section{Laplace operator}

We define the Laplace operator $\Delta: H(V) \rightarrow H(V)$ to be

Thus, we have

$$
\Delta f=-\frac{1}{2} \operatorname{div}(d f)(7)
$$

$$
(\Delta f)_{j}=d_{j} f_{j}-\sum_{i \sim j} w_{i j} f_{i}(8)
$$

The graph Laplacian is a linear operator. Moreover, the graph Laplacian is self-adjoint and positive semidefinite.

Let $S_{2}(f)=<\Delta f, f>$, we have the following theorem 1

$$
D_{f} S_{2}=2 \Delta f(9)
$$

The proof of the above theorem can be found from [6, 7].

\section{Curvature operator}

We define the curvature operator $\kappa: H(V) \rightarrow H(V)$ to be

Thus, we have

$$
\kappa f=-\frac{1}{2} \operatorname{div}\left(\frac{d f}{\|d f\|}\right)(10)
$$

$$
(\kappa f)_{j}=\frac{1}{2} \sum_{i \sim j} w_{i j}\left(\frac{1}{\left\|d_{i} f\right\|}+\frac{1}{\left\|d_{j} f\right\|}\right)\left(f_{j}-f_{i}\right)
$$

From the above formula, we have

$$
d_{i} f=\left((d f)_{i j}: j \sim i\right)^{T}(12)
$$

The local variation of $f$ at $i$ is defined to be

$$
\left\|d_{i} f\right\|=\sqrt{\sum_{j \sim i}(d f)_{i j}^{2}}=\sqrt{\sum_{j \sim i} w_{i j}\left(f_{j}-f_{i}\right)^{2}} \text { (13) }
$$

To escape the zero denominators in (11), the local variation of $f$ at $i$ is defined to be

$$
\left\|d_{i} f\right\|=\sqrt{\sum_{j \sim i}(d f)_{i j}^{2}+\epsilon},(14)
$$

where $\epsilon=10^{-10}$.

The graph curvature is a non-linear operator.

Let $S_{1}(f)=\sum_{i}\left\|d_{i} f\right\|$, we have the following theorem 2

$$
D_{f} S_{1}=\kappa f(15)
$$

The proof of the above theorem can be found from [6, 7].

\section{p-Laplace operator}

We define the p-Laplace operator $\Delta_{p}: H(V) \rightarrow H(V)$ to be

$$
\Delta_{p} f=-\frac{1}{2} \operatorname{div}\left(\|d f\|^{p-2} d f\right)(16)
$$

Thus, we have

$$
\left(\Delta_{p} f\right)_{j}=\frac{1}{2} \sum_{i \sim j} w_{i j}\left(\left\|d_{i} f\right\|^{p-2}+\left\|d_{j} f\right\|^{p-2}\right)\left(f_{j}-f_{i}\right)
$$

Let $S_{p}(f)=\frac{1}{p} \sum_{i}\left\|d_{i} f\right\|^{p}$, we have the following

theorem 3

$$
D_{f} S_{p}=p \Delta_{p} f(18)
$$

\section{Discrete regularization on graphs and credit cards' fraud transactions detection problems}

Given a transaction network $G=(V, E)$. $V$ is the collection of all transactions (i.e. nodes) in the graph (i.e. network) and $E$ is the collection of all potential relations between these transactions (i.e. nodes). Let $y$ represent the preliminary function in $H(V) . y_{i}$ can be defined as follows

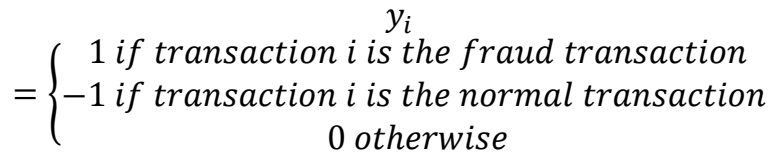

Our objective is to search for an approximated function $f$ in $H(V)$ such that $f$ is not only smooth on $G$ but also near enough to an preliminary function $y$. Then each transaction $i$ is classified as $\operatorname{sign}\left(f_{i}\right)$. This idea can be expressed as the subsequent optimization problem

$$
\operatorname{argmin}_{f \in H(V)}\left\{S_{p}(f)+\frac{\mu}{2}\|f-y\|^{2}\right\}(19)
$$

The first term in (19) is the smoothness term. The second term is the fitting term. A positive parameter $\mu$ captures the trade-off between these two competing terms.

\section{1) p-smoothness}

For any number $p$, the optimization

problem (19) is

$$
\operatorname{argmin}_{f \in H(V)}\left\{\frac{1}{p} \sum_{i}\left\|d_{i} f\right\|^{p}+\frac{\mu}{2}\|f-y\|^{2}\right\},
$$

By theorem 3, we have

satisfies

Theorem 4: The solution of (20)

$$
\Delta_{p} f+\mu(f-y)=0,(21)
$$

The $p$-Laplace operator is a non-linear operator; hence we do not have the closed form 
solution of equation (21). Thus, we have to construct iterative algorithm to obtain the solution. From (21), we have

$$
\begin{aligned}
& \frac{1}{2} \sum_{i \sim j} w_{i j}\left(\left\|d_{i} f\right\|^{p-2}+\left\|d_{j} f\right\|^{p-2}\right)\left(f_{j}-f_{i}\right)+ \\
& \mu\left(f_{j}-y_{j}\right)=0(22) \\
& \text { Define the function } m: E \rightarrow R \text { by } \\
& m_{i j}=\frac{1}{2} w_{i j}\left(\left\|d_{i} f\right\|^{p-2}+\left\|d_{j} f\right\|^{p-2}\right) \text { (23) } \\
& \text { Then equation (22) which is } \\
& \sum_{i \sim j} m_{i j}\left(f_{j}-f_{i}\right)+\mu\left(f_{j}-y_{j}\right)=0 \\
& \text { can be transformed into } \\
& \left(\sum_{i \sim j} m_{i j}+\mu\right) f_{j}=\sum_{i \sim j} m_{i j} f_{i}+\mu y_{j}(24) \\
& \text { Define the function } p: E \rightarrow R \text { by } \\
& p_{i j}=\left\{\begin{array}{l}
\frac{m_{i j}}{\sum_{i \sim j} m_{i j}+\mu} \text { if } i \neq j \\
\frac{\mu}{\sum_{i \sim j} m_{i j}+\mu} \text { if } i=j
\end{array}\right. \\
& \text { Then } \\
& f_{j}=\sum_{i \sim j} p_{i j} f_{i}+p_{j j} y_{j}(26) \\
& \text { Thus we can consider the iteration } \\
& f_{j}^{(t+1)}=\sum_{i \sim j} p_{i j}^{(t)} f_{i}^{(t)}+p_{j j}^{(t)} y_{j} \text { for all } j \in V \\
& \text { to obtain the solution of (20). }
\end{aligned}
$$

\section{Experiments and results}

\section{Datasets}

In this paper, we use the transaction dataset available from [9]. This dataset contains 284,807 transactions. Each transaction has 30 features. In the other words, we are given transaction data matrix $\left(R^{284807 * 30}\right)$ and the annotation (i.e. the label) matrix $\left(R^{284807 * 1}\right)$. The ratio between the number of fraud transactions and the number of normal transactions is 0.00173 . Hence we easily recognize that this is the imbalanced classification problem. In order to solve this imbalanced classification problem, we initially apply the under-sampling technique which is the Cluster Centroid technique [8] to this imbalanced dataset. Then the ratio between the quantity of fraud transactions and the quantity of normal transactions is 0.4 . In the other words, we are given the new transaction data matrix $\left(R^{1722 * 30}\right)$ and the annotation (i.e. the label) matrix $\left(R^{1722 * 1}\right)$.

Then we construct the likeness graph from the transaction data. The likeness graph used in this paper is the k-nearest neighbor graph: Transaction $i$ is connected with transaction $j$ if transaction $i$ is among the k-nearest neighbor of transaction $j$ or transaction $j$ is among the $\mathrm{k}-$ nearest neighbor of transaction $i$.

In this paper, the likeness function is the Gaussian likeness function

$$
s(T(i,:), T(j,:))=\exp \left(-\frac{d(T(i,:), T(j,:))}{t}\right)
$$

In this experiment, $t$ is set to 0.1 and the 5-nearest neighbor network is employed to build the likeness graph from the new transaction data.

\section{Experimental Results}

In this section, we test the above offered un-normalized graph p-Laplacian methods with $p=1,1.1,1.2,1.3,1.4$, $1.5,1.6,1.7,1.8,1.9$ and the current state of the art method (i.e. the un-normalized graph Laplacian based semi-supervised learning method $p=2$ ) in terms of classification accuracies. The accuracy $\mathrm{Q}$ is set in this way

$$
Q
$$

True Positive + True Negative

$=\overline{\text { True Positive }+ \text { True Negative }+ \text { False Positive + False Nega }}$ All trials were carried out in Matlab 6.5. The new transaction data is separated into two sub-collections: the training collection and the testing collection. The training collection contains 1,208 transactions. The testing collection contains 514 transactions. The parameter $\mu$ is set to 1 .

The accuracies of the above offered methods and the current state of the art method is specified in the subsequent table 1

\begin{tabular}{|c|c|}
\hline \multicolumn{2}{|c|}{$\begin{array}{c}\text { Accuracy Performance } \\
\text { Measures }(\%)\end{array}$} \\
\hline $\mathrm{p}=1$ & 88.52 \\
\hline $\mathrm{p}=1.1$ & 88.52 \\
\hline $\mathrm{p}=1.2$ & 88.52 \\
\hline $\mathrm{p}=1.3$ & 88.52 \\
\hline $\mathrm{p}=1.4$ & 88.52 \\
\hline $\mathrm{p}=1.5$ & 88.52 \\
\hline $\mathrm{p}=1.6$ & 88.52 \\
\hline $\mathrm{p}=1.7$ & 88.52 \\
\hline $\mathrm{p}=1.8$ & 88.52 \\
\hline $\mathrm{p}=1.9$ & 88.52 \\
\hline $\mathrm{p}=2$ & 88.33 \\
\hline
\end{tabular}

Table 1: The evaluation of accuracies of offered methods with diverse p-values

From the above table, we simply known that the unnormalized graph p-Laplacian semi-supervised learning methods beat the current state of art method. The outcomes from the above table illustrates that the unnormalized graph p-Laplacian semi-supervised learning methods are at least as decent as the current state of the art method $(p=2)$ but often produce better classification accuracies.

\section{Conclusions}

We have established the thorough regularization frameworks for the un-normalized graph p-Laplacian semi-supervised learning methods applying to the credit cards' fraud transactions detection problem. Trials illustrate that the un-normalized graph p-Laplacian semi-supervised learning methods are at least as decent 
as the current state of the art method (i.e. $p=2$ ) but often produce decisively better classification accuracies.

Sooner or later, we will construct the thorough regularization frameworks for the un-normalized hypergraph $\mathrm{p}$-Laplacian semi-supervised learning methods and will apply these methods to this credit cards' fraud transactions detection problem.

\section{Acknowledgement}

This research is funded by Vietnam National University Ho Chi Minh City (VNU-HCM) under grant number C2018-42-02.

\section{References}

[1] Goldstein, Markus, and Seiichi Uchida. "A comparative evaluation of unsupervised anomaly detection algorithms for multivariate data." PloS one 11.4 (2016): $\mathrm{e} 0152173$.

[2] Singh, Anshul, and Devesh Narayan. "A survey on hidden markov model for credit card fraud detection." International Journal of Engineering and Advanced Technology (IJEAT) 1.3 (2012).

[3] Nune, Ganesh Kumar, and P. Vasanth Sena. "Novel Artificial Neural Networks and Logistic Approach for
Detecting Credit Card Deceit." International Journal of Computer Science and Network Security (IJCSNS) 13.9 (2013): 58.

[4] Şahin, Yusuf G., and Ekrem Duman. "Detecting credit card fraud by decision trees and support vector machines." (2011).

[5] Shin, Hyunjung, Andreas Martin Lisewski, and Olivier Lichtarge. "Graph sharpening plus graph integration: a synergy that improves protein functional classification." Bioinformatics 23.23 (2007): 3217 3224.

[6] Tran, Loc Hoang, and Linh Hoang Tran. "Unnormalized graph p-Laplacian semi-supervised learning method applied to cancer classification problem." Journal of Automation and Control Engineering Vol 3.1 (2015).

[7] Tran, Loc, and Linh Tran. "The Un-normalized Graph p-Laplacian based Semi-supervised Learning Method and Speech Recognition Problem." Int. J. Advance Soft Compu. Appl 9.1 (2017).

[8] Yen, Show-Jane, and Yue-Shi Lee. "Cluster-based under-sampling approaches for imbalanced data distributions." Expert Systems with Applications 36.3 (2009): 5718-5727.

[9] https://www.kaggle.com/mlg-ulb/creditcardfraud 